\section{Malaria programmes need informed advocacy}

SIR - Your Editorial 'Time to take control' (Nature 451, 1030; 2008) seems to downplay the current importance of advocacy for maintaining funds in the fight against malaria. But funding for all elements of malaria programmes - from research and development, design, implementation and commodities, to monitoring and evaluation - is essential to success.

Results don't happen without dedicated and predictable financial resources and, although commitments have increased, we still have a long way to go. A recent study by McKinsey (see http://tinyurl.com/52aoda) estimates that an investment of some US $\$ 2.2$ billion a year for five years is needed to achieve full coverage of prevention and treatment measures in just 30 of the hardest-hit African countries where malaria is endemic, addressing $90 \%$ of current malaria deaths. Without continued pressure on donors to keep up their commitments, there is no guarantee that additional funding will ever materialize.

Also, advocacy is critical for ensuring that malaria efforts reach the communities most in need. On-the-ground efforts to engage national health leaders, navigate bureaucracies and encourage effective implementation of sound public-health policies are crucial. Without them, life-saving drugs, bed nets and insecticides will sit and spoil in warehouses. Generating and supporting participation and commitment on the spot is essential to the success of any programme.

The Editorial highlights one of the most extreme situations where informed advocacy is needed. In the Democratic Republic of the Congo, the first step in addressing the extremely high burden of malaria is fervent advocacy to increase investment in malaria programmes in challenging regions. Without that advocacy, government and donors will continue to avoid investment, and there will be no progress to measure.

Matthew Lynch

Global Program on Malaria,

Center for Communication Programs,

Johns Hopkins University, 111 Market Place,

Suite 310, Baltimore, Maryland 21202, USA

\section{Malaria: efforts starting to show widespread results}

SIR - Your Editorial 'Time to take control' misrepresents the role and work of the Roll Back Malaria (RBM) Partnership, and is apparently at odds with the successes described in the News Features and Commentary in the same issue (Nature 451, 1030, 1042-1049 and 1051-1052; 2008) .

The RBM Partnership, a coalition of hundreds of organizations, is an independent global public-health partnership that is governed by an international board. The board's members include the governments of countries where malaria is endemic, donor governments, bilateral and multilateral organizations, academia, civil society, the private sector and various foundations.

We agree that disease surveillance is essential for effective malaria control. Many RBM partners advocate more funding for this purpose. Indeed, RBM has provided the technical leadership and coordination to develop the approach and tools for national malaria indicator surveys that have provided valuable data to assess progress on coverage and impact.

We continue to advocate rapidly improving access to the bed nets and treatments that, every day, prevent unnecessary deaths. Strong advocacy is vital for surveillance to strengthen as prevention and treatment coverage expands.

Malaria control was severely underresourced until the first grants from the Global Fund to Fight AIDS, Tuberculosis and Malaria began to reach country programmes in 2004. New support - from the Bill and Melinda Gates Foundation, the US President's Malaria Initiative and the World Bank booster programme - further increased global resources, and nationwide scale-up of malaria control is now possible. However, much more funding is still needed.

Although many countries are still far from reaching RBM's target of $80 \%$ coverage, a growing number - including Benin, Cameroon, Eritrea, Ethiopia, Kenya, Madagascar, Mali, Niger and Rwanda have recently achieved significant progress in increasing access to long-lasting nets as a result of large-scale distribution campaigns carried out in the past four years.

Many countries have not yet conducted household surveys to record these higher coverage rates, but the improvements have been well documented (see, for example, http://tinyurl. com/3foako and http:// tinyurl.com/3oj3ey).

Results-oriented country support is a major feature of the partnership's work plan. It builds on 2007's successes, when the RBM Partnership led a strong campaign to help countries improve the quality of their proposals to the Global Fund. The success rate of proposals in Round 7 doubled from that of 2006, adding US\$471 million to fight malaria. Both Nigeria and the Democratic Republic of the Congo were prioritized at the last RBM board meeting in 2007 to receive additional implementation support from partners in 2008.

We believe that your Editorial does a disservice to our global efforts to turn back this preventable and treatable disease, at a time when we are seeing positive results.

Awa Marie Coll-Seck, Tedros Adhanom Ghebreyesus, Alan Court

RBM Partnership, Secretariat hosted by the World Health Organization, 20 Avenue Appia, CH-1211 Geneva 27, Switzerland

\section{Tibetan wildlife is getting used to the railway}

SIR - Your News story 'Acclaimed photo was faked' (Nature 451, 1034-1035; 2008) indirectly calls into question the impact of China's Qinghai-Tibet railway on the migration of Tibetan antelopes. As zoologists officially responsible for evaluating the impact of the railway on plateau wildlife, we have been monitoring the situation annually from the time construction began in 2003, through its completion in 2006 and on to the present.

During the railway's main construction period, the antelopes were very much disturbed. But they soon adjusted their migration routes, westward in spring and eastward in August, to avoid most human activity. The railway has many underpasses, including 143 that are more than 100 metres wide; these wide underpasses have a total width of 46 kilometres. Adding in the many smaller ones, underpasses encompass 259 kilometres of the antelopes' main range.

Contrary to any impression that might have been conveyed by the faking of the acclaimed photograph, the animals have readily adapted

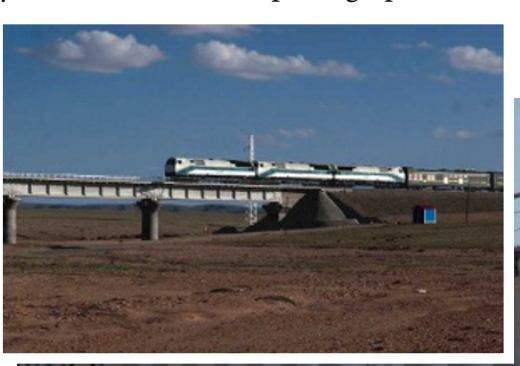
to these underpasses, at present concentrating on
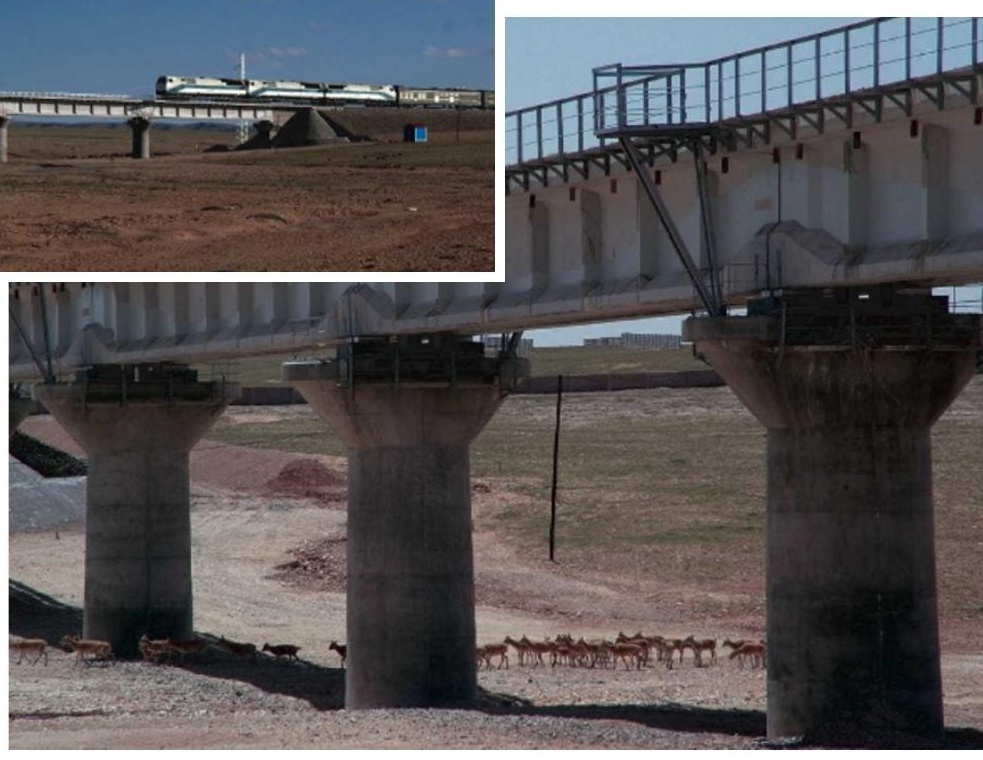

Building bridges? Migrating antelopes are not put off by the new railway. 